\title{
Seguridad de las vacunas y su repercusión en la población
}

\author{
Vaccine safety and its impact on the population
}

\section{Belkys María Galindo Santana, ${ }^{\mathrm{I}}$ Lellanis Arroyo Rojas, ${ }^{\text {II }}$ Damarys Concepción Díaz ${ }^{\text {III }}$}

I Doctor en Medicina. Máster en Epidemiología. Investigador Agregado. Instituto de Medicina Tropical "Pedro Kourí". La Habana, Cuba.

${ }^{\mathrm{II}}$ Licenciada en Estudios Socioculturales. Instituto de Medicina Tropical "Pedro Kourí". La Habana, Cuba.

IIIProgramadora de Sistemas. Instituto de Medicina Tropical "Pedro Kourí". La Habana, Cuba.

\section{RESUMEN}

La vacunación ha constituido uno de los logros más importantes en la historia de la salud pública y ha contribuido a la disminución de la morbilidad y mortalidad. A diferencia de los medicamentos, las vacunas se aplican generalmente a personas sanas para que continúen sanas, por tanto no deben ocasionar más daños que lo que podría producir la misma enfermedad. Es necesario que la población y la familia conozcan los beneficios y posibles riesgos que pudieran presentarse en el acto de vacunación, lo que proporcionaría mayor conocimiento sobre la importancia de las vacunas. Es tarea del médico enfatizar en este problema para garantizar mayor confiabilidad del programa de vacunación y seguridad en la población vacunada; debe, además, estar preparado para atender cualquier preocupación que surja en su área de salud o evento adverso que se produzca. El médico de familia, principal ejecutor de esta actividad, debe cumplir tres funciones básicas fundamentales: la educativa, la preventiva y la de vigilancia. En el futuro inmediato, las vacunas continuaran teniendo un gran impacto social en la prevención de las enfermedades infecciosas.

Palabras clave: Vacunas, población, eventos adversos, educación. 


\begin{abstract}
Vaccination is one of the most important achievements in the history of the public health and has contributed to the decrease of morbidity and mortality. Unlike the drugs, the vaccines are generally used in healthy persons for them to continue being healthy; therefore, they should not cause more damage than the disease as such. It is necessary that the population and the family know the benefits and possible risks of vaccination, which will expand their knowledge on the importance of vaccines. It is the duty of the physician to make emphasis on this problem in order to assure greater reliability of the vaccination program and its safety in the vaccinated population. He/she must be prepared to pay attention to any concern that may arise in the health area or to any adverse event that might occur. The family doctor as the main performer of this activity should fulfil three basic functions: educational, preventive and surveillance functions. In the near future, the vaccines will continue having great social impact on the prevention of the infectious diseases.
\end{abstract}

Key words: Vaccines, population, adverse events, education.

\title{
INTRODUCCIÓN
}

Durante los muchos años de la existencia del ser humano, la prevención de las enfermedades se intenta a través de medidas mágicas como el uso de amuletos, de conjuros o de diversas formas de actos de fe, a consecuencia de la ignorancia total sobre la etiología y la patogenia de las enfermedades. En ese largo período las actividades médicas solo pudieron enfocarse a la restauración de la salud pública mediante la búsqueda empírica de procedimientos o sustancias terapéuticas. ${ }^{1}$

En la historia de la salud pública algunas enfermedades de tipo infeccioso o carencial disminuyen ostensiblemente con la mejoría de las condiciones de vida, tales como la alimentación adecuada, disponibilidad de agua potable y medidas correctas para la eliminación de excretas, además de las mejorías experimentadas en el aspecto comunicativo, lo que produjo un aumento de la percepción de riesgo de las personas. Pero una de las medidas que mayor impacto tiene es la prevención de enfermedades infecciosas mediante programas organizados de vacunación los cuales han constituido uno de los logros más importantes, puesto que evitan muertes y enfermedades. Se le concede el honor al médico británico Edward Jenner, quien observa durante mucho tiempo que las lecheras de su pueblo (Berkeley) no enfermaban de viruela, por lo que deduce que podían haber padecido viruela vacuna, más leve que la humana y supuso que las protegía frente a esta. Inocula pus extraído de la pústula de una persona que padecía de viruela vacuna y días después la inocula con viruela humana y no desarrolla la enfermedad.

Todos estos resultados le permiten desarrollar la primera vacuna contra la viruela, cuya mortalidad en el Siglo XVIII es hasta del $40 \%$.

Cuando comienza la vacunación en el mundo contra esta terrible enfermedad, la incidencia de casos empieza a disminuir y alcanza su completa erradicación en 1977, y es ahí donde se reconoce el verdadero potencial de las vacunas. El mismo

http://scielo.sld.cu 
Jenner observa las primeras reacciones a esta vacuna que son las inflamaciones en el sitio de inoculación. ${ }^{1,2}$

\section{ANTECEDENTES}

La inmunización es el proceso de inducir artificialmente la inmunidad o proporcionar protección frente a una determinada enfermedad. La inmunización activa consiste en estimular al organismo para que produzca anticuerpos y otras respuestas inmunitarias a través de la administración de una vacuna, con el objetivo de que produzca una respuesta similar a la infección natural.

Una vacuna se define como una suspensión de microorganismos vivos, atenuados, inactivados o sus fracciones, administradas para inducir inmunidad y prevenir enfermedades infecciosas o sus secuelas. ${ }^{3}$

Las vacunas difieren de los medicamentos, por su naturaleza biológica, pues han sido creadas para prevenir enfermedades y se aplican por lo general a personas sanas, por tanto su fabricación, control y reglamentación exigen conocimientos y procedimientos especiales. Su distribución y comercialización se realiza a través de programas con estructuras sanitarias bien organizadas y además genera una vigilancia posterior a la vacunación que permite entregar información sobre eventos no pesquisados en los ensayos clínicos.

Durante los primeros años de la preparación y usos de las vacunas, su elaboración y control se comportó como un proceso totalmente artesanal. No hay métodos estandarizados para comprobar la pureza de las semillas bacterianas utilizadas, ni para valorar que la atenuación es suficiente y no siempre se hacen pruebas estrictas de esterilidad y con menos frecuencia se realizan las pruebas de potencia en animales, por lo que la mayoría de las veces, el producto se aplica directamente en humanos y solo se vigila la aparición temprana de reacciones adversas. ${ }^{1}$

Estas fallas de precaución son causas de graves desastres:

- En 1902 se utiliza en la India una vacuna contra la peste bubónica contaminada con Clostridium tetani y provoca la muerte por tétanos de 19 personas. ${ }^{1}$

- En 1930 en Alemania fallecen 75 lactantes después de ser vacunados con BCG, que contiene una cepa de Micobacterium tuberculosis. ${ }^{1}$

Para evitar estos desastres en Inglaterra se establecen las regulaciones de las sustancias terapéuticas con la intención de controlar la pureza, potencialidad y calidad de las sustancias inmunobiológicas.

A pesar de estas medidas, en 1955 ocurre un grave accidente en los laboratorios Cutter en los EE.UU. donde se aplicó un lote de vacuna de polio insuficientemente inactivada, que provoca casos de poliomielitis. ${ }^{1}$

Un paso fundamental para disminuir al máximo los riesgos de reacciones adversas consecutivas a la inmunización, es el establecimiento de los estudios de campo de las vacunas que contempla entre uno de sus requisitos la realización de estudios previos en voluntarios para demostrar que la preparación no es tóxica y que provoca pocas reacciones adversas a corto o largo plazo.

http://scielo.sld.cu 
En los momentos actuales la Organización Mundial de la Salud (OMS) ha establecido una serie de requisitos extraordinariamente rigurosos para la elaboración de las vacunas recogidos en: "Manual de Buenas Prácticas de Manufacturas" (BPM), lo cual ha contribuido a una notable disminución de las reacciones adversas posvacunales. ${ }^{1,4}$

La realidad es que las vacunas aportan más beneficios que riesgos, se aplican a personas sanas para que continúen siendo sanas, por tanto no deben ocasionar más daños que lo que podría producir la misma enfermedad y el riesgo de la presencia de algún evento adversos sea lo mínimo posible, ${ }^{1,4}$ debe ser eficaz y lograr una inmunidad protectora que sea perdurable en el tiempo. ${ }^{3}$

Con la disminución de la frecuencia de enfermedades, la población y los médicos van perdiendo la capacidad de percibir la importancia de la repercusión de las vacunas en la salud, así los eventos adversos comienzan a adquirir mayor importancia relativa, lo que hace poco tolerable para la opinión pública su aparición. Para ello es de gran importancia que la población conozca los beneficios y posibles riesgos que pudieran presentarse y es tarea del médico enfatizar en este problema, pues esto garantiza mayor confiabilidad del programa de vacunación y la seguridad de la población vacunada. ${ }^{4}$ Para que las vacunas protejan a todos, aproximadamente el 85 a $95 \%$ población debe estar inmunizados. Los estudiosos demuestran que los niños no inmunizados tienen más probabilidades de contraer enfermedades como sarampión o tos ferina. ${ }^{5}$

Es importante tener en cuenta que los primeros años de la vida de un niño es el período de mayor vulnerabilidad para adquirir enfermedades y se manifiestan trastornos del desarrollo, disminución de la capacidad auditiva, entre otros, y es en este momento donde se aplican la mayor cantidad de vacunas que muchas veces coincide con la aparición de estos trastornos, y es muy difícil determinar la verdadera causa.

En este período los principales responsables de la salud de sus hijos son justamente los padres, por esta causa se torna de vital importancia su convencimiento y entendimiento para proceder con la vacunación de sus pequeños. Al decir de García, la inmunización infantil ante determinadas enfermedades que pueden suponer un alto riesgo para la salud de los menores es un objetivo claro a nivel mundial. ${ }^{6}$

A pesar del importante avance en el control de las enfermedades, la inmunización no ha estado libre de controversias y existen algunos problemas de pérdida de credibilidad en la población, con consecuencias negativas para el mundo cuando se decide interrumpir la vacunación. Solo por mencionar un ejemplo, en el Reino Unido en la década del 70 del siglo pasado, consta una gran preocupación pública sobre los riesgos de las vacunas contra la tos ferina que conduce a una disminución en las coberturas de vacunación hasta de $30 \%$, lo que trae como consecuencia la presencia de más de 100000 casos, defunciones y hospitalizaciones y la ocurrencia de dos grandes epidemias evitables. Se requirió de un esfuerzo por parte de las autoridades de salud para realizar campañas educativas dirigidas a la población y de esta manera que se fuera recobrando lentamente la confianza en la vacuna y en su respectivo programa. Se elevan las coberturas al $95 \%$, por lo que se reduce de manera importante el número de casos, como se puede observar en la figura 1. 


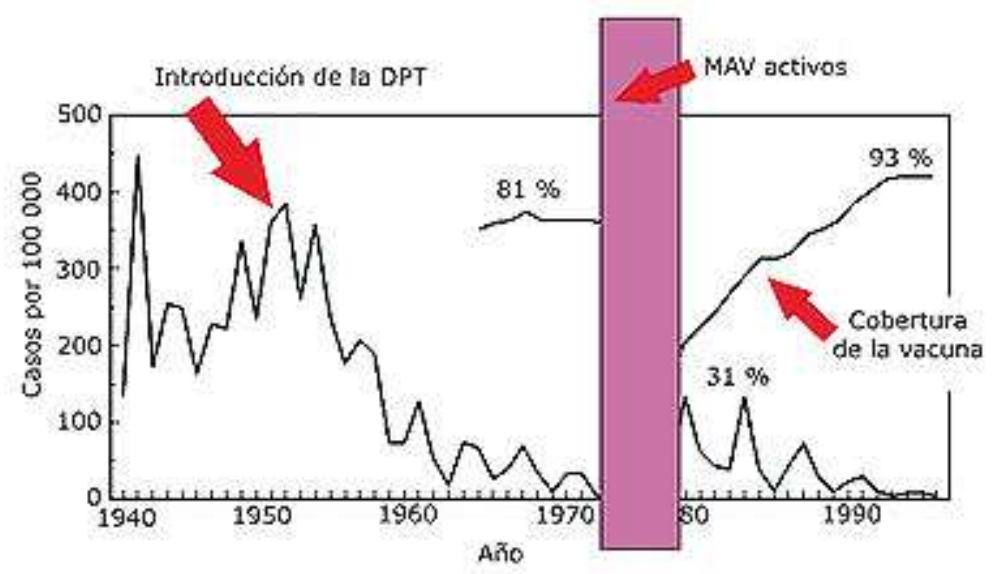

DPT: vacuna contra difteria, tetanos y tosferina, MAV: movimientos activos contra vacunas Fuente: Gangarosa. Lancet $351,1998$.

Fig. 1. Incidencia de la tos ferina en países afectados por los movimientos activos contra las vacunas - Inglaterra y Gales.

Las vacunas que se aplican en el ser humano, han pasado por varios procesos estrictos de calidad y deben tener una serie de requisitos como son: examen de las características de las vacunas, cumplimiento de las normas de buenas prácticas de fabricación, aprobación de la autoridad reguladora nacional que en el caso de Cuba, es el Centro para el Control Estatal de la Calidad de los Medicamentos (CECMED).

Las vacunas son más seguras que hace 40 años, pero no están exentas de riesgo de presentar algún evento adverso, que varía desde leve hasta eventos adversos graves que pueden conducir a hospitalizaciones, discapacidades, secuelas y hasta la muerte. Esto ocurre en un pequeño número de personas, por eso se requiere de un proceso educativo para la población y el personal de salud, el cual en caso de presentar un evento adverso debe actuar de forma inmediata, eficiente y con rigor científico para que se continúe reconociendo a la vacunación como uno de los logros más trascendentales de la salud pública mundial. ${ }^{7,8}$

\section{EVENTOS ADVERSOS}

El evento adverso a las vacunas es aquel accidente médico que ocurre después de la vacunación, causa preocupación y puede estar relacionado o no con la misma.

Según su expresión clínica estos pueden ser:

- Leves: son eventos locales, comunes y suelen ceder espontáneamente.

- Moderados: son aquellos que aunque requieren hospitalización, no dejan secuelas, ni incapacidad.

- Severos o graves: son aquellos que requieren hospitalización, ponen en riesgo la vida, dejan discapacidad o muerte.

Por tipo de asociación epidemiológica se pueden clasificar en: 
- Coincidente: ocurre después de la vacunación pero no causada por esta, es una posibilidad de asociación. Un ejemplo puede ser las crisis epilépticas.

- Reacción a la vacuna: (relación causal) debido a propiedades inherentes de la vacuna. Un ejemplo puede ser reacción a algunos componentes de la vacunas, dígase, timerosal.

- Error programático: debido a un error en preparación, manejo o administración de la vacuna. ${ }^{4}$

Especial importancia requieren los errores operativos o error programático (error humano), pues el vacunador puede prevenirlos en cualquier caso. Este error frecuentemente humano es causado por la vacuna o la tecnología. Por lo general las formas de prevención son: la capacitación y la supervisión y suministro apropiado de equipos para las inyecciones seguras. ${ }^{7}$

Las certezas de los siguientes errores pueden ayudar a identificar la causa de las reacciones adversas:

- Vacunas aplicadas en sitios incorrectos.

- Uso de agujas y jeringuillas no esterilizadas.

- Manipulación incorrecta de las agujas.

- Vacunas reconstituidas con diluentes no apropiados.

- Incremento de las dosis de vacuna.

- Sustitución de vacunas por otros productos.

- Vacunas y diluentes contaminados.

- Incorrecta conservación de vacunas.

- Congelar vacunas que contienen adyuvante de sales de aluminio.

- Ignorar contraindicaciones verdaderas.

- Utilizar frascos de vacuna abiertos e incorrectamente protegidos.

La figura 2 muestra los errores operativos y sus consecuencias. ${ }^{7}$ 


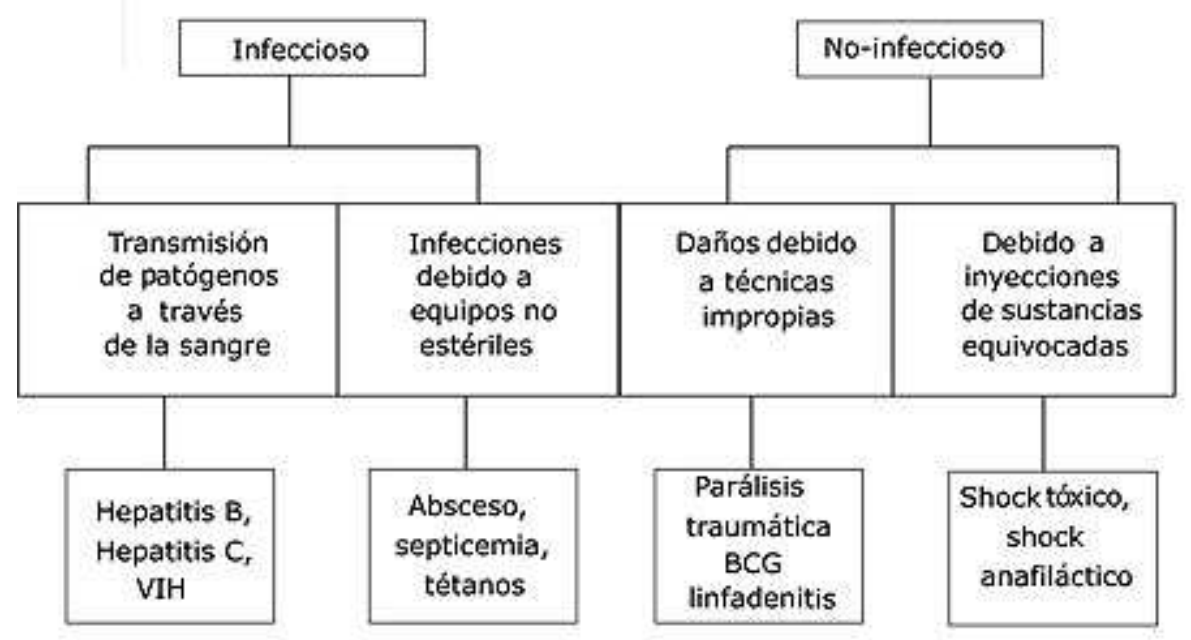

Fuente: Ob cit 7 ,

Fig. 2. Errores operativos del programa y las consecuencias que produce.

El error programático puede conducir a un conglomerado de eventos, especialmente si un vacunador no cumple con lo que se le enseña durante la capacitación. El caso más grave es el choque tóxico por la manipulación incorrecta del vial de la vacuna una vez reconstituido. Este caso podría traer como consecuencia que varios lactantes vacunados del mismo vial pueden morir poco tiempo después de la inyección.

Un buen funcionamiento de los sistemas de vigilancia vacunal debe ser una responsabilidad compartida del organismo de reglamentación farmacéutica y el programa nacional de inmunización. ${ }^{8}$

En Cuba existe un Programa Nacional de Inmunización (PNI), creado desde 1962, con gran impacto en la población cubana, pues ha permitido la eliminación de varias enfermedades, la eliminación de algunas formas clínicas y de complicaciones, que algunas enfermedades hayan reducido su morbilidad de forma significativa y otras hayan dejado de constituir un problema de salud. Por tanto, se requiere de un sistema de vigilancia de eventos adversos en el país que continuará permitiendo la confiabilidad del programa de vacunación cubano. Este sistema fue implementado en Cuba en enero de 1999 y ha permitido conocer la magnitud y trascendencia de los eventos adversos a la vacunación que era aún no bien valorada. Con la existencia del Programa se le da cumplimiento a un requisito exigido por el CECMED que es la identificación de los eventos más frecuentes y actuar de forma inmediata en el momento en que se presente un evento adverso para minimizar los riesgos. ${ }^{9-}$ 12

Los sistemas de vigilancia de eventos adversos a vacunas pueden ser:

- Vigilancia pasiva: a través de reportes espontáneos.

- Vigilancia activa: seguimiento activo posvacunal.

Es necesario llamar a la reflexión acerca de la importancia de lograr la inserción de la población en los distintos tipos de vigilancia. De este forma se potencializa aún más la comunicación entre el personal de salud y los destinatarios de sus servicios. 
La mayoría de los sistemas existentes en el mundo corresponden a sistema de vigilancia pasiva.

Varios países tienen implantado sistemas de vigilancia, tal es el caso de: Chile, EE. UU., México, Canadá; sistemas globales como en Noruega y Suiza y a partir de 2003 con el desarrollo tecnológico de nuevas vacunas en el mundo, la OMS y la Organización de Naciones Unidas para el desarrollo de la Infancia (UNICEF), crearon la red de seguridad de vacunas. ${ }^{13,14}$

Cuando se produce un evento adverso fatal en el proceso de vacunación conlleva a una crisis o a una situación donde hay pérdida real o potencial de confianza en las vacunas o en el servicio de vacunación, precipitada por información sobre un evento adverso (real o supuesto), que puede ser evitada mediante la previsión, cuidado y adiestramiento del personal de salud. Si el programa de vacunación está bien organizado se pueden reducir al mínimo los errores en el proceso de vacunación, para continuar fortaleciendo el programa de inmunización, por tanto, se requiere de mejor nivel de comunicación a los familiares, brindar los datos necesarios para que estén mejor informados sobre los beneficios y posibles riesgos que puedan presentarse. Se pretende que el personal de salud tenga la suficiente capacidad técnica para poder enfrentar este problema, poder identificar los eventos de forma precoz y adoptar las medidas oportunas lo más rápidamente posible. ${ }^{7}$

Tres funciones básicas deben cumplirse desde la atención primaria de salud en la vigilancia de los efectos adversos de las vacunas:

1. Función educativa: mediante la explicación a los padres y tutores de los beneficios que se derivan de la vacunación, las enfermedades que previenen y alertar de los posibles efectos secundarios que pueden aparecer.

2. Función preventiva: se realiza a partir del conocimiento de las reacciones adversas más frecuentes para su correcta identificación y tratamiento, de las contraindicaciones verdaderas para evitar posponer innecesariamente las inmunizaciones indicadas e identificación de los pacientes con mayor riesgo de reacciones adversas o graves. Asimismo, deben tener la formación adecuada para la identificación de reacciones anafilácticas y urgencias asociadas a la administración de vacunas potencialmente tratables y para su manejo pertinente.

3. Función de vigilancia activa o pasiva: mediante la detección de eventos adversos inesperados y comunicarlos a través de los programas de farmacovigilancia.

Cuando se va a introducir una nueva vacuna, su promoción debe orientarse a informar suficientemente al público en general para fomentar su confianza y mostrarle el impacto que se espera lograr con la vacuna en la prevención o en el control de la enfermedad en cuestión.

Los medios de comunicación masiva desempeñan una función importante en la formación de la percepción de la opinión pública sobre la vacunación y pueden tener una influencia positiva o negativa, por ello resulta fundamental la creación de alianzas con estos actores desde el inicio, para que se involucren en el desarrollo de la estrategia de comunicación.

La vigilancia de los eventos adversos de la nueva vacuna debe incorporarse al sistema de vigilancia de eventos adversos a la vacunación que existe en el país y reforzarlo haciendo énfasis en la vigilancia activa e intensificada de todos los 
eventos adversos descritos por el fabricante o de eventos inesperados. Estos procederes permitirán tomar oportunamente las medidas que fueran necesarias.

Finalmente se puede concluir que las vacunas han jugado un papel muy importante en la prevención de enfermedades infecciosas y que generalmente son inocuas y evitan morbilidad y mortalidad. En el futuro inmediato, las vacunas continuaran teniendo un gran impacto social devenido por la prevención de las enfermedades infecciosas. El personal de salud encargado de la actividad de vacunación debe estar capacitado para enfrentar cualquier evento adverso que se produzca y solucionarlo lo más rápido posible, vía que permite recobrar la confianza, credibilidad y fortalecimiento de los programas de vacunación. Un buen nivel de información a la familia sobre los beneficios de la vacunación y sus posibles riesgos ayudaran a aumentar el conocimiento sobre la importancia de las vacunas.

\section{AGRADECIMIENTOS}

A Yasel Manuel Santiesteban Díaz, estudiante de 5to. año de Psicología, por su valiosa ayuda.

\section{REFERENCIAS BIBLIOGRÁFICAS}

1. Escobar A, Valdespino JL, Sepúlveda J. Vacunas. Ciencia y salud. Historia de las vacunas y las vacunaciones. México: INDRE;1992.

2. CDC. Recomendations and Reports. (Smallpox). Vaccine, recomendations of the Advisory Comité on Immunization Practices (ACIP). MMWR. 2001;50(RR-10).

3. Moreno M, Amat J, Moya M, Cruz S. Reacciones adversas producidas por vacunas infantiles. Alergol Inmunol Clin. 2005;20:51-63.

4. Galindo MA, Galindo B, Pérez A, Santín M. Sistema de vigilancia de eventos adversos consecutivos a la vacunación. La Habana: Ministerio de Salud Pública, Dirección Nacional de Estadísticas;1999.

5. American Academy of Pediatrics. Información para padres acerca la inocuidad de las vacunas [sitio en Internet]. [actualizado Mar 2008; citado 7 Dic 2009]. Disponible en: http://aapredbook.aappublications.org/cgi/spanish_pdf/2003/1/1.3.pdf

6. García Y. ¿Vacunaciones obligatorias de menores contra la voluntad de los padres? Humanidades Médicas. 2009;(35):11-21.

7. OPS/OMS. Vacunación segura: como enfrentar eventos adversos supuestamente atribuidos a la vacunación e inmunización. Washington, D.C.: OPS/OMS;2002.

8. Vargas J. La seguridad de las vacunas: un tema importante para la población. Rev Perú Med Exp Salud Pública. 2006;23(1):2. 
9. OPS/MINSAP. Evaluación internacional del programa de inmunizaciones de Cuba. La Habana: OPS/MINSAP; 2004.

10. Ministerio de Salud Pública de Cuba (MINSAP). Programa Nacional de Inmunización. La Habana: MINSAP; 2004.

11. Galindo B, Galindo MA, Pérez A, Santín M. Diseño de un sistema de vigilancia de eventos adversos consecutivos a la vacunación en Cuba. Rev Cubana Med Trop. $1999 ; 51(3): 197-8$.

12. Galindo B, Berdasquera D. Vigilancia de eventos adversos a la vacunación: experiencia cubana en la atención primaria de salud. Rev Cubana Med Gen Integr [serie en Internet]. 2007[citado 7 Dic 2009];23(1). Disponible en:

http://scielo.sld.cu/scielo.php?script=sci arttext\&pid=S0864$\underline{21252007000100019 \& \operatorname{lng}=\mathrm{es}}$

13. Abarca K. Vigilancia de eventos adversos a vacunas. Rev Chil infect. $2007 ; 24$ (1):53-6.

14. Lankinen Kari S. Vigilancia de las vacunas en Europa: necesidad de acción rápida, normalización y recursos. Bull World Health Organ. 2004;82(11):828-35.

Recibido: 5 de febrero de 2010.

Aprobado: 26 de febrero de 2010.

Belkys María Galindo Santana. Instituto de Medicina Tropical "Pedro Kourí" (IPK). Autopista Novia del Mediodía Km 6²/2, La Lisa, Apartado postal 601, Marianao 13. La Habana, Cuba. Correo electrónico: bgalindo@ipk.sld.cu 